\title{
Seizure-induced neglect
}

\author{
KENNETH M HEILMAN AND GREGORY J HOWELL \\ From the Department of Neurology, College of Medicine, University of Florida, and the Veterans \\ Administration Medical Center, Gainesville, Florida, USA
}

SUMMARY A man with intermittent right parieto-occipital seizures was monitored by electroencephalography while he received 60 trials of being touched on the right, left, or both hands. Half of the trials were given during a focal seizure, and half were given interictally. While the patient was having seizures, he appropriately responded to all 10 stimuli delivered to the right hand, but four of 10 responses were incorrect (allaesthetic) when he was stimulated on the left. With bilateral simultaneous stimulation he neglected the left hand in all 10 trials. His interictal performance was flawless. When given a line-bisection task on two occasions during a seizure, the patient attempted to make a mark to the left of the entire sheet of paper. Immediately postictally he made a mark at the right end of the line. The case illustrates that focal seizures may induce elements of the neglect syndrome and that attention (to contralateral stimuli) and intention to perform (in the contralateral hemispatial field) may be dissociable phenomena.

Under a variety of stimulus conditions, patients who can detect stimuli fail to report, respond, or orient to stimuli presented on the side contralateral to a cerebral lesion. The failure to report or respond appropriately to unilateral stimuli has been termed "unilateral neglect" or "inattention." ${ }^{1}$ Sometimes patients with unilateral lesions respond appropriately to unilateral stimuli but fail to report the contralateral stimulus when stimulated on both sides simultaneously. Critchley ${ }^{1}$ thought that extinction to simultaneous stimulation is another instance of unilateral neglect made manifest at a particular moment by the technique of simultaneous stimulation. When touched on the side opposite to their lesion, patients with unilateral neglect often reported that they were touched on the side ipsilateral to their lesion. This has been termed "allaesthesia."2 Critchley ${ }^{1}$ thought that unilateral neglect also was responsible for allaesthesia.

Because inattention can be induced by a variety of cortical and subcortical lesions, we have postulated that many of the behavioural signs reflect an attention-arousal disorder induced by disruption of a corticolimbic reticular loop. ${ }^{34} \mathrm{We}$ have also postulated that some of the behaviour

Address for reprint requests: Dr KM Heilman, Box J-236, Department of Neurology, College of Medicine, University of Florida, Gainesville, FL 32610, USA.

Accepted 10 July 1980 seen in the neglect syndrome, such as hemispatial neglect, may be induced by an intentional defect. ${ }^{45}$ Recently, we observed a patient with right parietal lobe seizures. The patient showed that elements of the neglect syndrome may be associated with focal seizures. He also provided evidence for the postulate that attentional and intentional defects are dissociable.

\section{Case report}

A 63 year old man was in good health until two days before admission when he developed "impairment of vision in the left eye." He had had difficulty seeing the chain on a night light. He attributed the problem to impaired vision in the left eye because the vision in his right eye was already poor secondary to a cataract. He had no other specific complaints until the next morning when he began to have intermittent episodes of "double vision." During these episodes, everything seemed to move to the right and appeared to have constantly fluctuating colours. The patient did not complain of dizziness but did say that during the two days before admission he had difficulty in walking, which was most severe during the episodes of "double vision."

Past medical and family histories were noncontributory. Medication included spironolactone with hydrochlorothiazide and digoxin. Physical examination showed the patient's temperature to be $36.7^{\circ} \mathrm{C}$; pulse rate, 80 beats per min and regular; respirations, 16 per min and unlaboured; and blood pressure, $135 / 90 \mathrm{mmHg}$ without postural change. 
Neurological examination showed an alert but indifferent right-handed man who was oriented in time, place and person. He accurately named some of the preceding presidents and recalled major current events. Despite distraction, he was able to recall three objects five minutes after presentation, and he could recall seven numbers forward and four in reverse order. There was no aphasia, acalculia, finger agnosia, or right-left confusion. Reading and writing also were normal. The patient had difficulty drawing a cube, a square, and a triangle. He also appeared to have an indifferent affect. Several other tests of right-hemisphere function were performed. The patient's ability to judge mood from pictures of faces was incorrect ${ }^{6}$ in nine of 12 trials; he correctly identified only happy faces. In judging verbal mood, he was incorrect in five of 12 trials; he would judge what was said rather than how it was said. His ability to create a mood with his own voice was impaired except for happy moods. Song recognition and spontaneous singing were normal. When asked to tell whether two pictures of unfamiliar faces were the same or different, he made four errors in 12 trials.

The pupils were equal in size, round, and reacted to light and accommodation. Visual acuity in the left eye was 20/70 with glasses; the patient could see only hand movements with the right eye because of a dense cataract. There was a dense left homonymous visual field defect. The remainder of the cranial nerves were normal. Motor examination, including muscle strength, tone, and coordination, was normal except for a tendency for the outstretched left arm to rise. Cerebellar testing, including finger-to-nose, heel-to-shin, and rapid alternating movements, was normal. The patient walked cautiously and appeared to have slight bradykinesia of the left limbs. Deep tendon reflexes were normal except for absent ankle jerks. The right plantar response was flexor, and the left was equivocal. There were no other pathologic reflexes. Sensory examination, including pain, position, vibration, and graphesthesia, was normal except for mild impairment of vibration in the distal portion of both lower extremities.

During the neurological examination the patient complained that an episode of "double vision" was beginning. His head and eyes then began to deviate to the left. On command he could momentarily return his head and eyes to the right; however, they again promptly deviated to the left. The sensation of diplopia was present even with his eyes closed. During this seizure the previously noted tendency for the outstretched left arm to rise increased, and the patient was slow to respond to questions but answered appropriately. Because he had several components of the neglect syndrome, special testing was performed.

During an interictal period, when asked to place numbers on a clock, he incorrectly placed the numbers only on the right half of the clock. When given a map of Florida, he located the cities in the right half of the state. He would write his name in the right upper quadrant of a sheet of paper and would incorrectly bisect a line too far to the right even when he was able to identify correctly the left side of the line by searching for a letter at that end.5 His mood was generally euphoric, but when told he was having seizures, he adamantly denied he was having anything but brief spells of "double vision."

When we tested for auditory extinction, the patient was asked to close his eyes. In the unilateral conditions (unilateral left, unilateral right), the examiner simultaneously snapped the fingers of both the right and left hand approximately $5 \mathrm{~cm}$ from the patient's right or left ear. In the bilateral condition, the fingers of both hands were simultaneously snapped; however, one hand was placed behind each of the patient's ears. We randomised the order in which these three conditions were given. Tests for tactile extinction were performed in a similar manner. In the unilateral conditions, the back of the right or left hand was touched. In the bilateral condition both hands were simultaneously touched on the back. The order in which these conditions were given was randomised.

Interictally, the patient was given 18 auditory trials, six in each condition, and 24 tactile trials, eight each condition. He flawlessly performed all of these trials. While he was having a seizure, he was also given 18 auditory trials, six in each condition. He flawlessly detected and lateralised all unilateral stimuli; however, with bilateral simultaneous auditory stimuli, he neglected the left-side stimulus in all six trials. During a seizure he was also given 14 unilateral tactile stimuli (seven to each hand) and six bilateral stimultaneous tactile stimuli. With unilateral right-hand tactile stimuli, his performance was flawless. With unilateral left-hand tactile stimuli, he detected all seven stimuli, but on two of these trials he thought he was being touched on the right hand (allaesthetic response). With bilateral stimuli he neglected left-hand stimulation in all six trials.

An EEG performed on the day of admission disclosed occasional spikes and an occasional slow wave focus in the right parieto-occipital region. With the onset of the patient's sensation of diplopia, the record revealed polyspikes from the right parietooccipital area that gradually increased in amplitude and rhythm with a decrease in frequency to approximately two to three $\mathrm{Hz}$ sharp and slow wave discharge that became generalised before returning to baseline rhythms (see figure).

While being monitored with the EEG, the patient received 60 trials of being touched on the right, left, or both hands. Half of the trials were given during a seizure and half were given interictally. The stimulus conditions were randomised. While he was having a seizure, he correctly recognised all 10 stimuli delivered to the right hand, but when he was touched on the left hand, four of 10 responses were incorrect (allaesthetic). With bilateral simultaneous stimulation he neglected the left hand in all 10 trials. 


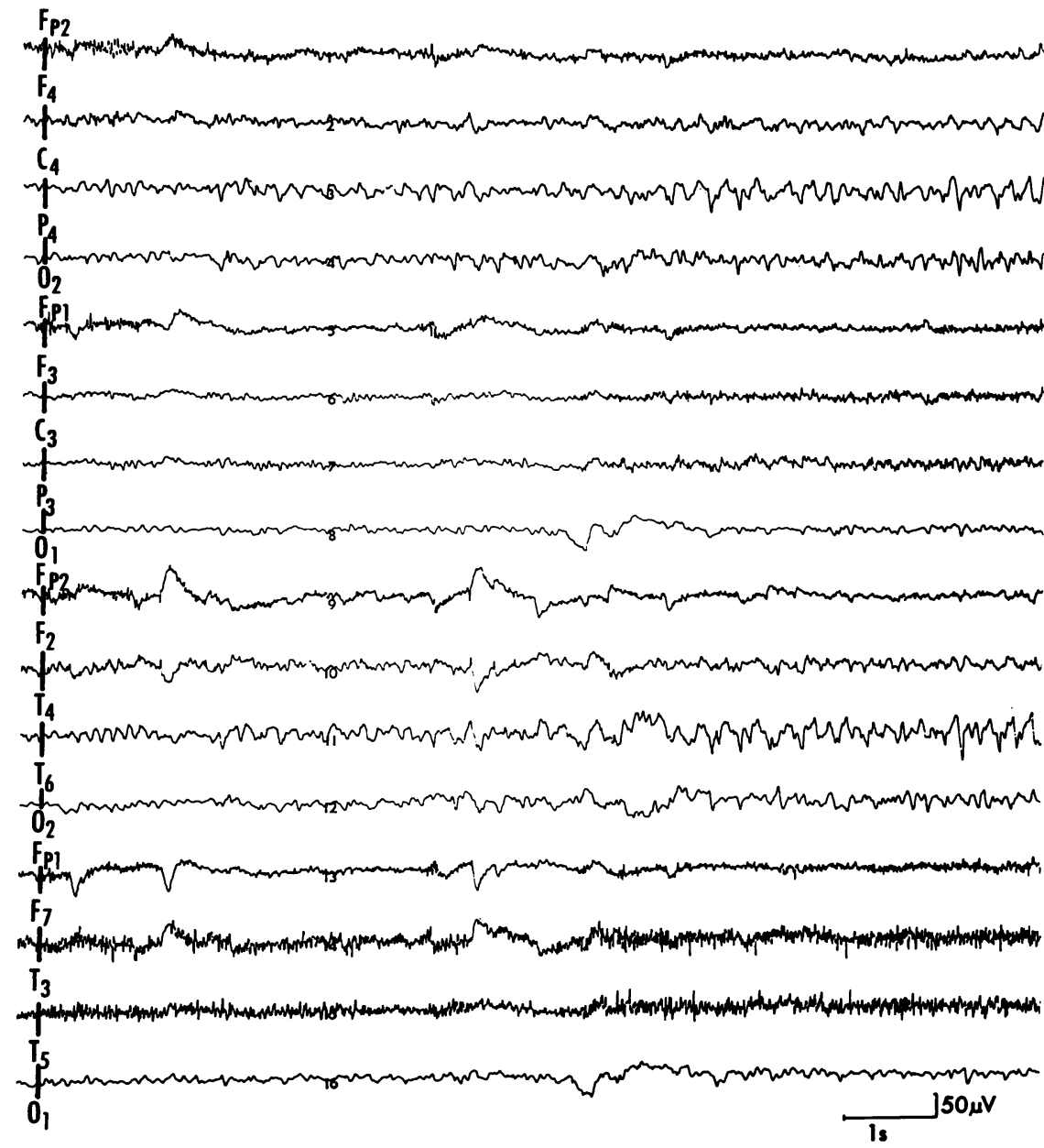

Fig Electroencepha-

logram recorded at onset of focal seizure.

His interictal performance was flawless. When given a line bisection task, on two occasions during a seizure, he had difficulty seeing the line; but when encouraged to bisect the line on the sheet of paper directly in front of him, he attempted to make a mark beyond the left edge of the entire sheet. Immediately postictally, he made a mark at the right end of the line. Several minutes later, although he continued to make a mark to the right side of the line, his mark was more accurate. Also immediately after a seizure, he performed a crossing-out task, and he failed to cross out two of the 12 marks. The two he missed were on the left side. During an interictal interval he took the same test and crossed out all lines. The extinction and allaesthetic phenomena disappeared with cessation of the electrographic seizure. Immediately postictally, however, he was momentarily mildly hypokinetic with a blunted affect.

Other pertinent laboratory values included a normal haematocrit, white blood count, platelets, and erythrocyte sedimentation rate. The Veneral Disease Research Laboratory test for syphilis was nonreactive. Abnormal test results included: fasting glucose $11.9 \mathrm{mmol} / \mathrm{l}$; serum cholesterol, $9.6 \mathrm{mmol} / \mathrm{l}$; and triglyceride, $23.7 \mathrm{mmol} / \mathrm{l} ;$ and lipoprotein electrophoresis showed Type $\mathrm{V}$ hyperlipoproteinemia. The electrocardiogram and chest and skull X-ray films were normal. A brain scan revealed increased uptake in the right parieto-occipital area. A fourvessel cerebral angiogram revealed evidence of luxury perfusion in this same area. Computed tomography of the brain did not reveal any abnormality.

We had difficulty controlling the patient's partial seizures with diphenylhydantoin, and he required subsequent addition of phenobarbitone and carbamazepine; the three medications controlled the seizures. On the third seizure-free day and two days before discharge, he was again tested for hemispatial 
neglect at a time when the visual fields were normal. The line bisection and crossing-out tasks were correctly performed. He could correctly number a clock, draw an entire daisy, and draw a cube. Mapping was improved.

\section{Discussion}

This patient manifested many symptoms of the neglect syndrome during and after a focal seizure. During a seizure he had allaesthesia to left-side tactile stimulation, and left-side extinction to bilateral auditory and tactile stimuli. During a seizure, however, when asked to bisect a line, he tended to bisect the line farther to the left than the left edge of the paper.

We assess patients with hemispatial neglect by holding a card in front of them and centring it at the midline of their body. We then ask them to close their eyes and to touch the card directly opposite their midline. Patients with left hemispatial neglect often point to the right of midline. We have never seen a patient who has pointed beyond the left edge of the sheet. We therefore cannot attribute this man's intention to bisect the line beyond the left of the card during a seizure to a decrease in visual acuity during the seizure. Immediately postictally he again incorrectly bisected the line but this time toward the right of midline; his interictal performance was also to the right but was more accurate.

The seizure focus appeared to be emanating from the right parietal lobe. The importance of the parietal lobe in attentional processes can be inferred not only from ablation studies in man and monkeys ${ }^{5}$ but also from intracellular recording in performing animals. Yin and Mountcastle ${ }^{7}$ and Lynch et al, ${ }^{8}$ using single cortical cell recordings, identified in the parietal lobe a class of neurons that had large receptive fields and were activated by light presented in the contralateral half field. There was a subclass of cells that enhanced their firing if the stimulus was significant and was followed by movement. The parietal "attentional" cells that these workers ${ }^{7} 8$ described performed a function similar to that which Sokolov ${ }^{9}$ proposed for his cortical comparator neurons. Robinson et al ${ }^{10}$ also demonstrated the presence of attentional cells in the parietal lobes. These cells not only responded to significant visual stimuli with enhanced firing but also responded to somaesthetic stimuli.

It appears that during our patient's seizures these attentional cells could not function as comparators and that dysfunction of these attentional cells was responsible for the manifestations of inattention. Although we consider both allaesthesia and extinction as manifestations of inattention, these are different behaviours; consequently, the brain mechanisms underlying these behaviours must differ. The difference underlying these behaviours is possibly related to how other portions of the brain respond while the attentional cells are improperly functioning.

When patients with a stable lesion, such as cerebral infarction, recover from neglect, they go from a stage in which they are inattentive to unilateral stimuli or have allaesthetic responses, to a stage in which they can detect and correctly lateralise unilateral stimuli but neglect contralesion stimuli with bilateral simultaneous stimulation. ${ }^{14}$ Although while having a seizure, this patient neglected all the auditory and tactile stimuli delivered to the left during bilateral simultaneous stimulation, when undergoing unilateral stimulation he made no errors in the auditory modality but did make allaesthetic errors in the tactile modality. We have noted a similar pattern in other patients with neglect. Inattention, allaesthesia, and extinction of somaesthetic and visual stimuli are more severe and recover more slowly than these symptoms in the auditory modality. We are not certain why this happens. Although the auditory system has stronger contralateral than ipsilateral projections, the auditory system has more ipsilateral hemispheric afferents than do either the somaesthetic or the visual systems. It is also possible that there are parietal "attention cells" similar to those described by Lynch et al, ${ }^{8}$ which "attend" to auditory stimuli. Based on our observation, we would suspect that unlike the visual attention cells, which are activated by visual stimuli mainly in the contralateral visual half field, these auditory cells would be more likely to be activated by ipsilateral stimuli.

Pribram and McGuiness ${ }^{11}$ proposed that attention-arousal, which is concerned with stimulus reception, and intention, which is a readiness or preparation for action, were independent but interrelated processes. After training animals to move an extremity contralateral to a stimulus, Watson et $a l^{12}$ induced neglect with dorsolateral frontal and intralaminar thalamic lesions. When stimulated on the side contralateral to their lesion, the animals performed normally; but when stimulated on the nonneglected side (ipsilateral to the lesion), they failed to use the extremity opposite the lesion despite normal strength. Valenstein, Van Den Abell, and Heilman ${ }^{13}$ have found a similar 
phenomenon in animals with parietotemporal lesions.

Heilman and Valenstein ${ }^{5}$ proposed that the unilateral hypokinesia may not be limited to the extremity contralateral to the lesion but is a hypokinesia to any stimulus that comes to the hypoaroused and hypoactivated hemisphere. In the case of hemispatial neglect, Heilman and Valenstein ${ }^{5}$ proposed that each hemisphere is responsible not only for mediating movements of the contralateral extremity and processing contralateral sensory input, but also for mediating behaviour in the contralateral spatial field independent of which extremity is used. This hemispatial hypokinesia may not be limited to the extremities but may also include eye movements.

Studies of normal subjects support the hypothesis that each hemisphere is important for mediating behaviour in the contralateral spatial field. The time taken to react to a lateralised visual stimulus is determined by the anatomical connections between the receiving hemiretina and the responding hand. Ipsilateral responses, which are mediated by intrahemispheric neuronal circuits, are faster than contralateral responses, which require interhemispheric transfer. ${ }^{14}$ For example, if a stimulus is presented to the right visual field, the response is faster with the right hand than with the left. This is no longer the case in choice reaction times when the arms are crossed so that the right hand is on the left side of the body and the left hand is on the right side. In this situation, stimuli in the right visual field for example are responded to faster with the left hand than with the right.

This phenomenon has been termed "stimulus response compatibility"15 and has been thought to reflect a natural tendency to respond with the hand that is already in the appropriate hemispatial field. An alternative explanation is that each hemisphere is responsible not only for moving the contralateral extremity and processing contralateral stimuli but also for mediating behaviour in the contralateral hemispatial field, regardless of which extremity is being used. The right hand, for example, takes longer to respond to a right-sided stimulus when it is in the left hemispatial field than when it is in the right hemispatial field. This is because, in the former instance, bilateral hemispheric processing is required: the left hemisphere must process the visual stimulus, but the right hemisphere must contribute to the response of the right hand because it is in the left hemispatial field. $H$ bisect lines incorrectly because hemianopia or sensory hemiinattention prevents them from see ing how far the line extends to the left, a strategy that ensures their seeing the left side of the line in their normal field should improve performance. If patients have hemispatial hypokinesia, moving the line toward the normal half of body space should improve performance.

Heilman and Valenstein ${ }^{5}$ tested patients with hemispatial neglect by requiring them to identify a letter at either the right or the left end of a line before bisecting that line. The task was given with the lines placed at either the right, the centre, or the left of the body midline.

Performance in trials when subjects were required to look to the left before bisecting a line did not differ from when they were required to look right. Performance was significantly better when the line was placed to the right side of the body than to the left. These observations support the hypothesis that patients with hemispatial neglect have hemispatial hypokinesia.

Although the results of this study are compatible with the hemispatial hypokinesia hypothesis, there is an alternative explanation. William James" ${ }^{16}$ noted that "an object once attended will remain in the memory whilst one inattentively allowed to pass will leave no trace behind." Although our subjects saw the full extent of the line, it is possible that the side of the line in the left hemispatial field did not form a stable trace. As the subject explored the remainder of the line, he "forgot" the left side of the line and performed as if he had not seen it. The hemispatial field is not the same as the visual field but refers to the space to one side of the midline of the body. If there were an attentional hemispatial memory defect, it would not be for the portion of the line in the left visual field but rather for the portion of the line on the left side of the body. Unfortunately, Heilman and Valenstein's ${ }^{5}$ experimental paradigm could not distinguish between the hemispatial hypokinesia and hemispatial inattention-memory hypotheses; however, if one accepts the latter hypothesis, it is difficult to explain why patients with hemispatial neglect draw half a daisy when a spontaneously drawn daisy requires no afferent input.

Because attention and intention are closely linked, the brain mechanisms underlying intention are not well known. In man, disease of the basal ganglia and frontal lobes may induce defects that are predominantly intentional. Although the inferior parietal lobe has direct projection to the frontal lobe, ${ }^{17}$ we suspect that the mesencephalic and thalamic reticular systems 
and limbic systems are also important in mediating intention. In this patient the localised right parietal seizure appeared to activate these intentional systems, not only inducing eye and head deviation to the left but also inducing the patient to incorrectly bisect lines to the left of the test sheet. Immediately after the seizure ended, the patient appeared to attend to contralateral stimuli; however, he then bisected lines to the right of centre. Since Todd's ${ }^{18}$ description of postictal paralysis, many other postseizure defects have been described (for example, aphasia, sensory loss); however, the postictal hemispatial neglect demonstrated by this man has not been recognised. The mechanism underlying postictal changes has not been completely elucidated, and we do not know why an intentional defect persisted after the seizure terminated. However, the postictal hemispatial neglect in the absence of inattention helped us to further substantiate what was seen during the seizure-namely, that defects of intention and attention can be dissociated.

This study was supported in part by National Institutes of Health Grant NS-12218. Dr LJ Willmore helped us with the EEG, and Mrs Alice W Cullu provided editorial assistance.

\section{References}

1 Critchley M. The Parietal Lobes. New York: Hafner Press, 1966.

2 Obersteiner H. On allochiria-a peculiar sensory disorder. Brain 1882; 4:153-63.

3 Heilman KM, Watson RT. Mechanism underlying the unilateral neglect syndrome. $A d v$ Neurol 1977; 18:93-106.

4 Heilman KM. Neglect and related disorders. In: Heilman KM, Valenstein E, eds. Clinical Neuropsychology. New York: Oxford University Press, 1979; 268-307.

5 Heilman KM, Valenstein E. Mechanisms underlying hemispatial neglect. Ann Neurol 1979; 5:
$166-70$

6 DeKosky ST, Heilman KM, Bowers D, Valenstein E. Recognition and discrimination of emotional faces and pictures. Brain Lang (in press).

7 Yin TCT, Mountcastle VB. Visual input to the visuomotor mechanism of the monkey's parietal lobe. Science 1977; 197:1381-3.

8 Lynch JC, Mountcastle VB, Talbot WH, Yin TCT. Parietal lobe mechanisms of directed visual attention. J Neurophysiol 1977; 40:362-89.

9 Sokolov YN. Perception and the Conditioned Reflex. Oxford: Pergamon Press, 1963.

10 Robinson DL, Goldberg ME, Stanton GE. Parietal association cortex in the primate: sensory mechanisms and behavioral modulations. J Neurophysiol 1978; 41:910-32.

11 Pribram KH, McGuinness D. Arousal activation and effort in the control of attention. Psychol Rev 1975; 182:116-49.

12 Watson RT, Miller BD, Heilman KM. Nonsensory neglect. Ann Neurol 1978; 3:505-8.

13 Valenstein E, Van Den Abell T, Heilman KM. Nonsensory neglect from parietotemporal lesions in monkeys. Presented at the International Neuropsychological Society Meeting, February 2, 1980, San Francisco, California.

14 Berlucchi G, Heron W, Hyman R, et al. Simple reaction times of ipsilateral and contralateral hand to lateralized visual stimuli. Brain 1971; 94:419-30.

15 Anzola GP, Bertoloni A, Buchtel HA, et al. Spatial compatibility and anatomical factors in simple and choice reaction time. Neuropsychologia 1977; 15:295-302.

16 James W. The Principles of Psychology, vol 2. New York: Holt, 1890.

17 Pandya DM, Kuypers HGJM. Cortico-cortical connections in the rhesus monkey. Brain Res 1969; 13:13-36.

18 Todd RB. Clinical Lectures on Paralysis, Disease of the Brain and Other Affections of the Nervous System. Philadelphia, 1855. Cited by Meyer JS, Portnoy HD. Post-epileptic paralysis. A clinical and experimental study. Brain 1959; 82:162-85. 